\title{
Prevalência da retinopatia diabética no Ambulatório de Endocrinologia Pediátrica da Santa Casa de Misericórdia de São Paulo
}

\author{
The prevalence of diabetic retinopathy at the Pediatric Endocrinology \\ Outpatient Clinic of the Santa Casa de São Paulo
}

\author{
Daniel de Souza Pereira ${ }^{1}$ \\ Frederico Augusto Costa Reis ${ }^{1}$ \\ Ricardo Themudo Lessa Waetge ${ }^{2}$ \\ Luis Eduardo Caliari ${ }^{3}$ \\ Teruo Aihara $^{4}$
}

\begin{tabular}{|c|}
\hline RESUMO \\
\hline $\begin{array}{l}\text { Objetivo: Verificar a prevalência de retinopatia diabética encontrada na } \\
\text { população de diabéticos tipo } 1 \text {, acompanhada no Ambulatório de Endocri- } \\
\text { nologia Pediátrica da Santa Casa de Misericórdia de São Paulo, entre } 29 \text { de } \\
\text { agosto de } 2000 \text { e } 17 \text { de agosto de } 2001 \text {, e sua relação com o sexo, a idade do } \\
\text { paciente na ocasião dodiagnóstico do diabete e a duração do diabete. Método: } \\
\text { Foi realizado mapeamento de retina, biomicroscopia de fundo e retinografia } \\
\text { em } 81 \text { pacientes, } 41 \text { do sexo feminino e } 40 \text { do masculino, a fim de detectar e } \\
\text { classificar a retinopatia diabética nessa população. Resultados: A idade dos } \\
\text { pacientes variou entre } 4 \text { e } 23 \text { anos (média }=12,0 \pm 12,0) \text {, a idade do paciente } \\
\text { na ocasião do diagnóstico, entre } 6 \text { meses e } 15 \text { anos (média }=3,4 \pm 5,8) \text { ea } \\
\text { duração dodiabete, entre } 7 \text { meses e } 20 \text { anos }(\text { média }=5,8 \pm 4,4) \text {. Aretinopatia } \\
\text { diabética foi observada em } 14 \text { pacientes }(17,3 \%), 7(8,6 \%) \text { coma forma não } \\
\text { proliferativa muito leve, } 5(6,2 \%) \text { com forma não proliferativa leve, } 1(1,2 \%) \\
\text { com forma proliferativa de alto risco e } 1(1,2 \%) \text { com forma proliferativa } \\
\text { avançada. Conclusões: A prevalência da retinopatia diabética na nossa } \\
\text { amostra é } 17,3 \% \text {. Não há diferença entre os portadores e não portadores de } \\
\text { retinopatia diabética quanto a sexo e idade do paciente na ocasião do } \\
\text { diagnóstico. Quanto maior a duração da diabete, maior a prevalência da } \\
\text { retinopatia diabética. }\end{array}$ \\
\hline
\end{tabular}

Descritores: Retinopatia diabética/epidemiologia; Diabetes melittus insulino dependente; Prevalência; Hospitais comunitários; Brasil.
Trabalho realizado no Departamento de Oftalmologia da Santa Casa de Misericórdia de São Paulo.

${ }^{1}$ Fellow de Retina e Vítreo do Departamento de Oftalmologia da Santa Casa de São Paulo.

${ }^{2}$ Chefe do Serviço de Retina e Vítreo do Departamento de Oftalmologia da Santa Casa de São Paulo.

${ }^{3}$ Professor Assistente da Unidade de Endocrinologia do Departamento de Pediatria da Santa Casa de São Paulo.

${ }^{4}$ Professor Doutor, Assistente do Serviço de Retina e

Vítreo do Departamento de Oftalmologia da Santa Casa de São Paulo.

Endereço para correspondência: Rua General Jardim, 630/42, São Paulo (SP) CEP: 01223-010

E-mail: drdaniel pereira@hotmail.com

Recebido para publicação em 28.03.2003

Versão revisada recebida em 06.08.2003

Aprovação em 17.09.2003

Nota Editorial: Pela análise deste trabalho e por sua anuência na divulgação desta nota, agradecemos ao Dr. Pedro Paulo de Oliveira Bonomo.

\section{INTRODUÇÃO}

A retinopatia diabética é uma das principais causas de cegueira na Europa e nos Estados Unidos ${ }^{(1-2)}$. É a maior responsável por perda visual e cegueira em adultos profissionalmente ativos entre 20 e 64 anos nas sociedades economicamente desenvolvidas ${ }^{(3)}$. Calcula-se que o risco de cegueira nos diabéticos seja vinte e cinco vezes maior do que na população normal e que aproximadamente $19 \%$ dos casos de cegueira no mundo sejam causados por diabetes mellitus ${ }^{(1-2)}$. De toda a população mundial, estima-se que $0,5 \%$ seja afetada pelo diabetes mellitus tipo 1 e $10 \%$ pelo tipo $2^{(4)}$. No Brasil está entre as principais causas de cegueira irreversível, atingindo 7,6\% da população segundo o Ministério da Saúde ${ }^{(5)}$, sendo responsável por 4,58\% das deficiências visuais ${ }^{(6)}$.

As alterações do fundo do olho que ocorrem com a progressão da doença seguem um curso que se inicia na forma não proliferativa podendo 
evoluir para a forma proliferativa. Elas são resultados da microangiopatia que afeta o organismo do diabético ${ }^{(4)}$, caracterizada, nos estágios iniciais, por microaneurismas e oclusões vasculares e, nos estágios tardios, por formação de cicatrizes $^{(7)}$.

Tempo de duração da doença, descontrole glicêmico, hipertensão arterial sistêmica e lesões renais são reconhecidos como agravantes para o aparecimento da retinopatia diabéti$\mathrm{ca}^{(8)}$. A redução da hiperglicemia e da hipertensão arterial com manutenção em níveis normais e estáveis resulta em diminuição importante na progressão da retinopatia diabética proliferativa $^{(9)}$.

Vários estudos demonstram variações consideráveis na prevalência da retinopatia diabética, de 20 a $79 \%$, porém tentativas de avaliação das possíveis causas dessas variações ainda são inconcludentes ${ }^{(10)}$. O desenvolvimento da doença ocular é a maior preocupação do paciente diabético ${ }^{(11)}$. A prevalência da retinopatia cresce gradualmente com o tempo de duração da doença e, após 20 anos, quase todos os pacientes com diabetes mellitus tipo 1 mostram anormalidades nos vasos da retina ${ }^{(7)}$

\section{OBJETIVO}

O objetivo deste estudo foi verificar a prevalência de retinopatia diabética nos diabéticos do tipo 1, acompanhados no Ambulatório de Endocrinologia Pediátrica da Santa Casa de São Paulo, entre 29 de agosto de 2000 e 17 de agosto de 2001, e sua relação com o sexo, idade do paciente na ocasião do diagnóstico da diabete e duração da diabete.

\section{MÉTODOS}

Foi realizado estudo prospectivo dos diabéticos tipo1 acompanhados no Ambulatório de Endocrinologia Pediátrica da Santa Casa de São Paulo, no período de 29 de agosto de 2000 e 17 de agosto de 2001, totalizando 81 pacientes. Foi feito mapeamento de retina com lente asférica de 20 dioptrias, biomicroscopia do fundo do olho com lente asférica de 78 dioptrias e retinografia do pólo posterior com retinógrafo Topcon TRC-50X e filme Ectacrome 400.

As alterações retínicas encontradas foram estadiadas segundo a classificação do $\operatorname{ETDRS}^{(12)}$.

Os pacientes foram classificados levando-se em consideração o olho que apresentou mais alterações fundoscópicas após exame cuidadoso. Foram analisados: sexo, idade, idade do paciente na ocasião do diagnóstico da diabete, duração da diabete, presença e tipo de retinopatia diabética e necessidade de tratamento oftalmológico.

Para a análise dos resultados, foram aplicados os seguintes testes não paramétricos:

1- Teste do qui-quadrado para tabelas $2 \times 2$, com a finalidade de comparar, apenas no grupo de portadores de retinopatia, os sexos feminino e masculino ${ }^{(13)}$.

2- Teste do qui-quadrado para tabelas $5 \times 2$, com o objetivo de comparar, separadamente os grupos de portadores e não portadores de retinopatia em relação à idade do paciente na ocasião do diagnóstico da diabete e duração da diabete ${ }^{(13)}$.

Em todos os testes, fixou-se em 0,05 o nível para a rejeição da hipótese de nulidade.

\section{RESULTADOS}

Dos 81 pacientes examinados, 41 eram do sexo feminino $(51,0 \%)$ e 40, do masculino (49,0\%). A idade variou entre 4 e 23 anos (média $=12,0 \pm 12,0$ ), a idade do paciente na ocasião do

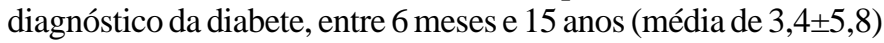
e a duração do diabetes, entre 7 meses e 20 anos (média = $5,8 \pm 4,4)$.

Não houve diferença entre os sexos quando avaliados quanto à presença de retinopatia (Tabela 1).

A idade do paciente na ocasião do diagnóstico da diabete não influenciou a presença de retinopatia (Tabela 2).

A duração da diabete teve relação direta com a presença de retinopatia (Tabela 3 ).

Não houve correlação entre o sexo e o tipo de retinopatia na nossa amostra (Tabela 4).

\section{COMENTÁRIOS}

Uma das complicações crônicas mais temidas do diabetes mellitus tipo 1(DM1) é a retinopatia ${ }^{(11)}$. Desde o início da doença as alterações glicêmicas podem propiciar alterações funcionais e anatômicas que evoluem para a retinopatia diabética $^{(6)}$. A prevalência desta complicação em pacientes com

Tabela 1. Pacientes diabéticos, segundo o sexo e a presença de retinopatia diabética. Santa Casa de São Paulo 2000/2001

\begin{tabular}{|c|c|c|c|c|}
\hline \multirow[t]{2}{*}{ Sexo } & \multicolumn{2}{|c|}{ Retinopatia } & \multirow[t]{2}{*}{ Total } & \multirow{2}{*}{$\begin{array}{c}\% \text { de } \\
\text { presença }\end{array}$} \\
\hline & Presença & Ausência & & \\
\hline Feminino & 9 & 32 & 41 & 22,0 \\
\hline Masculino & 5 & 35 & 40 & 12,5 \\
\hline Total & 14 & 67 & 81 & 17,3 \\
\hline \multicolumn{5}{|c|}{$\begin{array}{l}\text { Teste do qui-quadrado: } X^{2} \text { calculado }=1,26 \text { NS; } X^{2} \text { crítico }=3,84 ; \text { NS = Não } \\
\text { Significante }\end{array}$} \\
\hline
\end{tabular}

Tabela 2. Pacientes diabéticos, segundo idade de início do diabete $e$ presença de retinopatia diabética. Santa Casa de São Paulo 2000/2001

$\begin{array}{lcccc}\begin{array}{l}\text { Idade de } \\ \text { qdiagnóstico }\end{array} & \text { Presença } & \text { Ausência } & \text { Total } & \begin{array}{c}\% \text { de } \\ \text { presença }\end{array} \\ 0-13 & 5 & 11 & 16 & 31,2 \\ 3-16 & 3 & 19 & 22 & 13,6 \\ 6-15 & 3 & 20 & 23 & 13,0 \\ 9-12 & 3 & 14 & 17 & 17,6 \\ 12-15 & 0 & 3 & 3 & 0 \\ \text { Total } & 14 & 67 & 81 & 17,3 \\ \text { Teste do qui-quadrado: } X^{2} \text { calculado }=3,26 \mathrm{NS} ; \mathrm{X}^{2} \text { crítico }=9,49 ; \mathrm{NS}=\mathrm{Não} \\ \text { Significante }\end{array}$




\begin{tabular}{|c|c|c|c|c|}
\hline $\begin{array}{c}\text { Tempo de } \\
\text { diagnóstico }\end{array}$ & Presença & Ausência & Total & $\begin{array}{c}\% \text { de } \\
\text { presença }\end{array}$ \\
\hline $0-4$ & 1 & 40 & 41 & 2,4 \\
\hline $4-8$ & 5 & 13 & 18 & 27,8 \\
\hline $8 \dashv 12$ & 1 & 12 & 13 & 7,7 \\
\hline $12-16$ & 6 & 2 & 8 & 75,0 \\
\hline $16-20$ & 1 & 0 & 1 & 100,0 \\
\hline Total & 14 & 67 & 81 & 17,3 \\
\hline
\end{tabular}

Tabela 4. Pacientes diabéticos com retinopatia diabética, segundo o sexo e o tipo de retinopatia. Santa Casa de São Paulo 2000/2001

$\begin{array}{lcc}\text { Tipo de retinopatia } & \begin{array}{c}\text { Sexo feminino } \\ \mathbf{n = 9}\end{array} & \begin{array}{c}\text { Sexo } \\ \text { masculino } \\ \mathbf{n}=\mathbf{5}\end{array} \\ \text { RpDNP muito leve } & 7 & 0 \\ \text { RpDNP leve } & 1 & 4 \\ \text { RpDP de alto risco } & 0 & 1 \\ \text { RpDP avançada } & 1 & 0 \\ \text { Total } & 9 & 5 \\ \text { Não analisável. } & & \\ \text { RpDNP=Retinopatia diabética não proliferativa; RpDP = Retinopatia diabética } \\ \text { proliferativa }\end{array}$

DM1 é variável, dependendo de fatores como tempo de doença, idade do paciente e controle metabólico ${ }^{(8)}$. Neste estudo avaliamos o desenvolvimento da retinopatia em pacientes acompanhados no Ambulatório de Endocrinologia Pediátrica, com o objetivo de avaliar a prevalência em um grupo com menos tempo de diabetes e idade cronológica mais baixa.

A incidência de diabete de $51,0 \%$ no sexo feminino e $49,0 \%$ no masculino é semelhante ao descrito em outros estudos, não havendo aumento de risco de doença de acordo com o sexo ${ }^{(14)}$.

Encontramos uma prevalência de $17,3 \%$ de retinopatia diabética na nossa amostra. Destes, $64,3 \%$ são do sexo feminino e $35,7 \%$ do masculino. Contudo, ao comparar os sexos em relação à presença de retinopatia, não houve diferença significante.Outros estudos sugerem menor risco de desenvolvimento de retinopatia no sexo feminino ${ }^{(10)}$, fato não encontrado em nossos pacientes.

A amostra avaliada foi de pacientes de baixa faixa etária ao diagnóstico, 3,4 anos, mais precoce que de outros estudos. Kalter-Leibovici e col. ${ }^{(14)}$ observaram a média da idade do paciente na ocasião do diagnóstico do diabetes mellitus, de 9,5 anos. Contudo, em relação à idade do paciente na ocasião do diagnóstico, a análise estatística não mostrou diferença significante entre os sexos, não evidenciando, também, diferença entre os grupos de portadores ou não portadores de retinopatia.

Após 12 anos de duração da diabete, $77,7 \%$ dos pacientes do nosso trabalho apresentavam algum tipo de retinopatia, em concordância com estudos que reportam presença de retinopatia em aproximadamente 60 a $80 \%$ dos pacientes com mais de
10 anos de evolução da doença ${ }^{(15-16)}$, discordando, no entanto do trabalho de Bognetti e col. ${ }^{(11)}$ que observaram o mesmo em $17,0 \%$ dos pacientes da sua amostra.

$\mathrm{Na}$ análise da duração da diabete houve diferença significante entre os grupos de presença e ausência de retinopatia quando comparados em relação ao tempo de diagnóstico da diabete o que coincide com a literatura, que constata relação direta entre o tempo de diagnóstico da doença e a presença de retinopatia ${ }^{(8,10)}$.

Chama a atenção o fato de encontrarmos um paciente com menos de 4 anos de evolução do diabetes apresentar retinopatia. A recomendação é que a avaliação oftalmológica seja feita após 5 anos do diagnóstico.

\section{CONCLUSÕES}

Neste estudo conclui-se que:

1- A prevalência da retinopatia diabética no Ambulatório de Endocrinologia Pediátrica da Santa Casa de Misericórdia de São Paulo foi 17,3\%.

2- Não houve diferença entre os portadores e não portadores de retinopatia diabética quanto a sexo e idade do paciente na ocasião do diagnóstico da diabete.

3- Quanto maior a duração da diabete, maior a prevalência da retinopatia diabética.

\section{ABSTRACT}

Purpose: To verify the prevalence of diabetic retinopathy found in a type I diabetic population, accompanied at the Pediatric Endocrinology Outpatient Clinic of the "Santa Casa de Misericórdia de São Paulo", between August 29, 2000 and August 17, 2001, and it's relationship with sex, the patient's age on occasion of the diagnosis of diabetes and the duration of diabetes. Methods: Retinal mappings, biomicroscopic fundus and retinography were accomplished in 81 patients, 41 female and 40 male, in order to detect and to classify the diabetic retinopathies in that population. Results: The patients' age varied between 4 and 23 years (average $=12.0 \pm$ 12.0 ), the patients' age on occasion of the diagnosis, between 6 months and 15 years (average $=3.4 \pm 5.8$ ) and the duration of the diabetes, between 7 months and 20 years (average $=5.8 \pm$ 4.4). Diabetic retinopathy was observed in 14 patients (17.3\%), in $7(8.6 \%)$ with very mild and non-proliferative form, in $5(6.2 \%)$ with mild and non-proliferative form, in $1(1.2 \%)$ with high risk proliferative form and in $1(1.2 \%)$ with advanced proliferative form. Conclusions: The prevalence of diabetic retinopathy in our sample is $17.3 \%$. There is no difference between those with and those without of diabetic retinopathy regarding sex and the patient's age on occasion of the diagnosis. The longer the duration of the diabetes, the higher the prevalence of diabetic retinopathy.

Keywords: Diabetic retinopathy/epidemiology; Diabetes melittus, insulin-dependent; Prevalence; Hospital, community; Brazil 


\section{REFERÊNCIAS}

1. Klein R, Klein BE, Moss SE, Davis MD, DeMets DL. The Wisconsin epidemiologic study of diabetic retinopathy. III. Prevalence and risk of diabetic retinopathy when age at diagnosis is 30 or more years. Arch Ophthalmol 1984;102:527-32.

2. Klein R, Klein BE, Moss SE, Davis MD, DeMets DL. The Wisconsin epidemiologic study of diabetic retinopathy II. Prevalence and risk of diabetic retinopathy when age at diagnosis is less than 30 years. Arch Ophthalmol 1984;102:520-26.

3. Cunha Vaz J. Lowering the risk of visual impairment and blindness. Diabet Med 1998;15(Suppl 4):S47-50.

4. Souza EC, Esteves JF, Broilo VR. Retinopatia diabética não proliferativa. In: Retina e vítreo-clínica e cirurgia. Abujamra M, Ávila M, Barsante C, Farah ME, Gonçalves JOR, Lavinski J, Moreira Jr CA, Nehemy MB, Suzuki H, editores. São Paulo, Roca; 2000. p.485-98.

5. Ministério da Saúde, Sociedade Brasileira de Diabete. Diabete melito. Guia básico para diagnóstico e tratamento. Programa Harvard/ Joslin/ SBD. Brasília, 1996.

6. Schellini SA, Silva MRBM, Silva MAM. Diabetes, retinopatia diabética e cegueira. J Bras Med 1994;67:171-4.

7. Olk RJ, Lee CM. Classification of diabetic retinopathy, In: Diabetic retinopathy $11^{\text {a }}$ edição. Olk RJ, Lee CM editors. Philadelphia, J.B. Lippincott; 1993. p.3-20.

8. Roy MS. Diabetic retinopathy in African Americans with type I diabetes: The New Jersey 725: II. Risk factors. Arch Ophthalmol 2000;118:105-15.
9. Klein R, Klein BE, Moss SE, Cruickshanks KJ. The Wisconsin Epidemiologic Study of Diabetic Retinopathy: XVII. The 14-year incidence and progression of diabetic retinopathy and associated risk factors in type I diabetes. Ophthalmology 1998;105:1801-15.

10. Larsson LI, Alm A, Bergeinheim T, Lithner F, Bergstrom R. Retinopathy in diabetic patients aged 15-50 years in the county of Umea, Sweden. Acta Ophthalmol Scand 1999;77:430-6.

11. Bognetti E, Calori G, Meschi F, Macellaro P, Bonfanti R, Chiumello G. Prevalence and correlations of early microvascular complications in young type I diabetic patients: role of puberty. J Pediatr Endocrinol Metab 1997;10:587-92.

12. Classification of diabetic retinopathy from fluorescein angiograms.. ETDRS report number 11. Early Treatment Diabetic Retinopathy Study Research Group. Ophthalmology 1991;98(5suppl):807-22.

13. Siegel S, Castelan NJJ. Nonparametric Statistics Second Edition. Mc GrawHill. Int. ed.1988; p.399. Não localizado.

14. Kalter-Leibovici O, Leibovici L, Loya N, Kremer I, Axer-Siegel R, Karp M, Laron Z. The development and progression of diabetic retinopathy in type I diabetic patients: a cohort study. Diabet Med 1997;14:858-66.

15. Orchard TJ, Dorman JS, Maser RE, Becker DJ, Drash AL, Ellis D, LaPorte RE, Kuller LH. Prevalence of complications in IDDM by sex and duration. Pittsburgh Epidemiology of Diabetes Complications Study II. Diabetes 1990; 39:1116-24.

16. D'Antonio JA, Ellis D, Doft BH, Becker DJ, Drash AL, Kuller LH, Orchard TJ. Diabetes complications and glycemic control. The Pittsburgh Prospective Insulin - Dependent Diabetes Cohort Study Status Report after 5 yr of IDDM. Diabetes Care 1989;12:694-700.

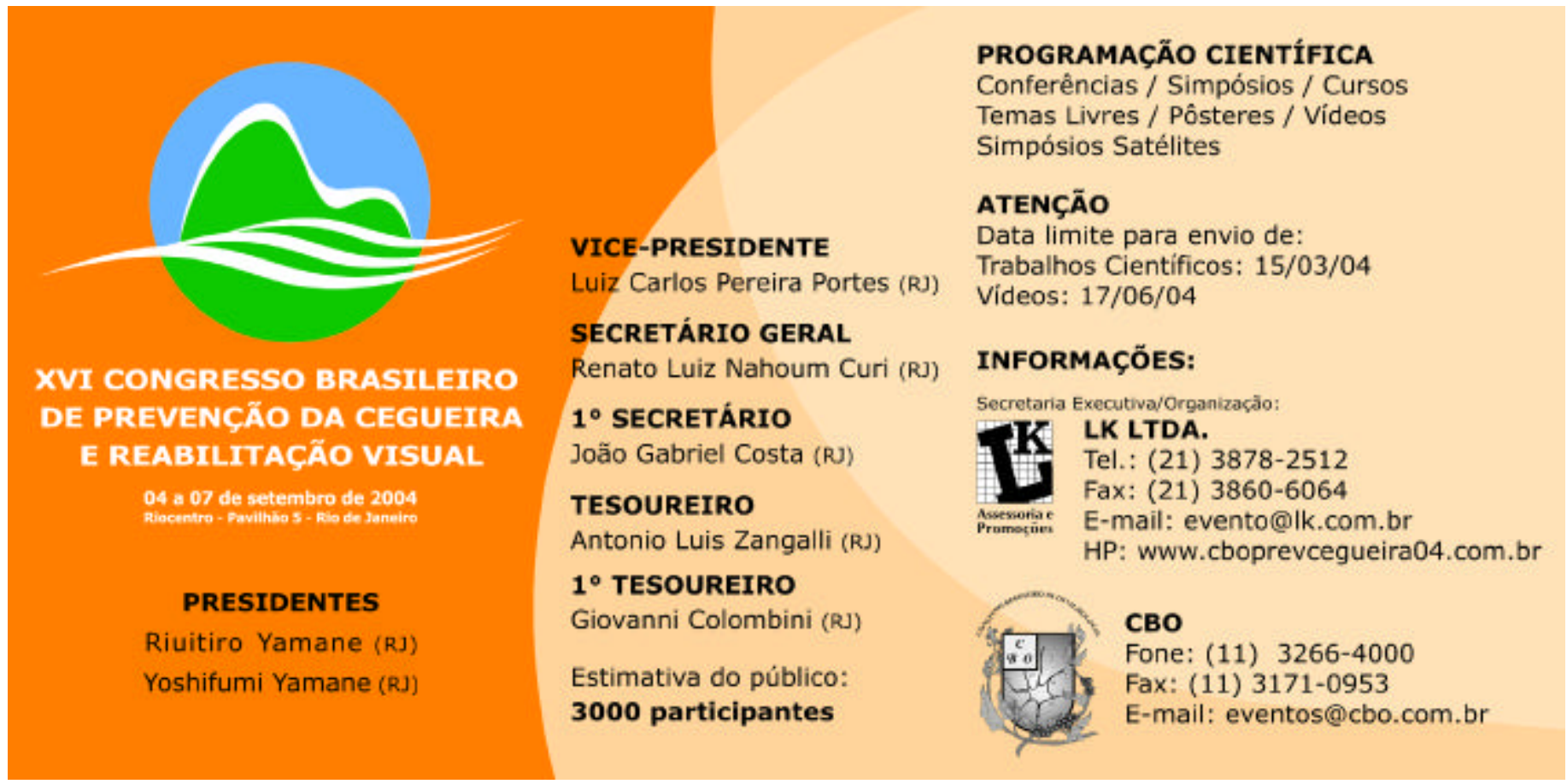

